\title{
Comments on the Discovery of a Ninth Planet
}

Wickramasinghe $\mathrm{NC}^{1,2^{*}}$

${ }^{1}$ Buckingham Centre for Astrobiology (BCAB), Buckingham University, UK

${ }^{2}$ Institute for the Study of Panspermia and Astroeconomics, Gifu, Japan

*Corresponding author: Wickramasinghe NC, Buckingham Centre for Astrobiology (BCAB), Buckingham University, UK, Tel: +44-777-838-9243; E-mail: ncwick@gmail.com

Recieved date: January 31, 2016; Accepted date: February 02, 2016; Published date: February 04, 2016

Copyright: (c) 2016 Wickramasinghe NC. This is an open-access article distributed under the terms of the Creative Commons Attribution License, which permits unrestricted use, distribution, and reproduction in any medium, provided the original author and source are credited.

\section{Editorial}

Planet X, a dwarf star binary companion to the Sun, and several variants of such ideas have been proposed over many years. The new report of a ninth planet, coming in the wake of the infamous Nibiru scam, may have escaped attention but for the credentials of its authors [1]. Batygin and Brown [1] (Brown played a key role in demoting Pluto from its planetary status) have argued for the existence of a Neptunesized planet in a highly elliptical orbit as the new ninth planet. The proposed new planet has a period of 20,000 years with a perihelion $200 \mathrm{AU}$ and an aphelion 600-1200AU (1AU=the Earth's mean distance from the sun). This claim is based on a peculiar clustering of orbital elements of 6 minor planets beyond Neptune's orbit $[1,2]$ that can be readily explained on the basis of the ninth planet. These "minor planets", including the dwarf planet Sedna and a body called 2012VP113 orbiting beyond Pluto, may all have been pulled into their highly elliptic orbits by the "ninth", as yet unseen, giant planet. In the absence of such a planet the observed orbital configurations of these smaller bodies could happen only with an exceedingly low probability.

If the Batygin-Brown planet does exist there remains a problem to understand how the planet was formed in a region of the solar nebula so far away from the orbits of Uranus and Neptune, the region where icy planetisimals (comet-like bodies) were thought to exist. One possible scenario is that it formed in the same region along with Uranus and Neptune but later came to be dynamically scattered into its present highly elliptic and more distant orbit.

Alternatively, the ninth planet, along with Sedna, and other Sednalike bodies may have been ripped off another star's planetary system as the star approached the sun to within a few hundred astronomical units. This is the capture mechanism proposed by Jilkova et al. [3] for the origin of Sedna based on results of their N-body numerical simulations. They proposed that such a capture event may have taken place early in the solar system's history, possibly in an encounter with a star within the birth cluster from which the sun itself was born.

From an astrobiological point of view, the interest in a ninth planet that accumulated from comet-like bodies is that it could be a major reservoir of microbial life in the solar system. Even more interesting would be the possibility that its life legacy was robbed from another star in the birth cloud of the solar system. The final proof of the existence of a ninth planet will of course be its actual telescopic detection.

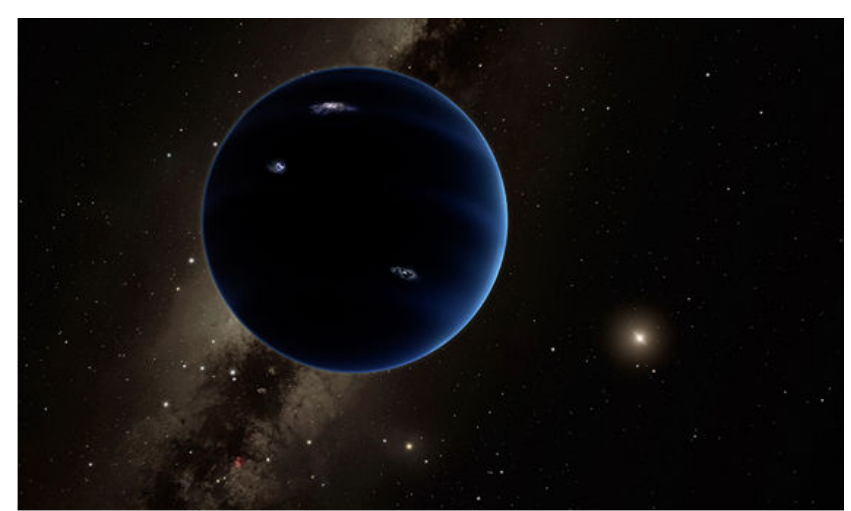

Figure 1: An artist's rendering shows the distant view from Planet Nine back towards the sun. The planet is thought to be similar to Uranus and Neptune. Hypothetical lightning lights up the night side. Credit: Caltech/R. Hurt (IPAC).

\section{References}

1. Batygin K, Brown ME (2016) Evidence for a Distant Giant Planet in the Solar System. The Astronomical Journal 151: 22.

2. Trujillo CA, Sheppard SS (2014) A Sedna-like body with a perihelion of 80 astronomical units. Nature 507: 471-474.

3. Jilkova L, Zwart SP, Pijloo T, Hammer M (2015) How Sedna and family were captured in a close encounter with a solar sibling. MNRAS 453: 3157-3162. 